\title{
The theory and applications of CRISPR in plant and tree improvement
}

\author{
Jack D. Parsons ${ }^{1}$ and John MacKay ${ }^{*}$ \\ Address: ${ }^{1}$ Department of Plant Sciences, University of Oxford, South Parks Road, Oxford, United Kingdom, \\ OX1 3RB
}

Correspondence: John MacKay, Email: john.mackay@plants.ox.ac.uk

\begin{abstract}
Increasing global population combined with a changing climate is placing pressure on global plant production systems, and rapid leafy and woody plant improvement is required to meet the demands of the future. Conventional breeding is time consuming, especially in woody plants, and potential new plant traits are limited by the pre-existing genetic diversity. CRISPR/Cas genome editing technology allows rapid, simple and targeted modifications of plant genomes, as well as the introduction of novel genes into the germplasm. Both agrobacterium and ribonucleoprotein (RNP) mediated delivery of CRISPR/Cas components to plant cells can produce transgene free edited plants, with F1 segregation required for the former. RNP mediated editing in protoplasts can be directly used for gene editing without transgenes, and for pyramiding of desired genes into germplasm. However, plant regeneration from protoplasts remains to be established for many woody species. In addition, high rates of SNP occurrence in outcrossing species, as well as a shortage of functional genomics knowledge, increases the complexity of designing CRISPR/Cas editing pipelines. However, despite some challenges, CRISPR/Cas genome editing has seen widespread application in the generation of disease resistant plants (providing resistance to fungal, bacterial and viral pathogens), as well as improving responses to abiotic stresses and increasing yields, amongst others. With the rapid development of new computational tools for designing and targeting CRISPR/Cas systems, increasing functional genomics knowledge, progress in protoplastRNP-based editing and favourable regulatory outlook, CRISPR/Cas genome editing is close to providing a new generation of future-proofed woody and leafy plants.
\end{abstract}

Keywords: Genome editing, biotic stress, abiotic stress, ribonucleoproteins, protoplasts, transgene-free, regulation

Review Methodology: Literature was selected based on its relevance to recent advances in CRISPR/Cas-mediated genome editing in leafy and woody crop plants. Key search terms included: 'CRISPR/Cas crops', 'CRISPR/Cas trees', 'CRISPR/Cas disease resistance', 'CRISPR/Cas transgene-free' and 'protoplast genome editing'. Multiple reviews on CRISPR/Cas mechanisms have been written, and the focus here is directed towards the theory and methodology of in planta genome editing, challenges faced with CRISPR/Cas use in plant, and generation of biotic and abiotic stress tolerance across leafy and woody species. 


\section{Background/Introduction}

By the year 2050, the global population is expected to increase from the current $\sim 7.6$ billion, to over 9 billion [1], placing additional strain on the earth's natural systems [2]. Woody plants play a crucial role in maintaining the stability of earth systems by limiting greenhouse gas emissions, retaining water (and so preventing flooding) and preserving diverse ecosystems, among others [2]. Trees such as eucalypt, poplar, and pines are widely planted and may provide both economic and ecological value. However, biotic and abiotic stresses, as well as lack of functional genomics understanding, remain limitations on tree genetic engineering and sustainable forestry [3], and the same issues are widely applicable across woody plant species. In order to sustain a rapidly growing population, it has been estimated that global agricultural output from woody and leafy plants must increase by between 60 and $110 \%$ [1]. However, comprehensive analysis suggests that, at the present rate, the world's four most important staple crops (rice, wheat, maize and soybean) will see output increases of $50.5 \%$ on average [4], well below what will likely be required. Increasing urbanisation is predicted to result in a net loss of global cropland of $1.8-2.4 \%$ by 2030 [5], meaning that expansion of arable land areas to meet plant production output requirements will not be possible. Therefore, an important approach to ensuring global agricultural security is likely to be increasing yields from currently available land. Such a strategy could be realised through increasing traits such as disease resistance [6], climate change tolerance [7] and photosynthetic efficiency [8], among others, in cultivated plants and trees.

Conventional breeding has largely relied on selection of pre-existing genetic variation in a plant species in order to generate a desirable germplasm [9]. Whilst the age of genomics has facilitated an increase in breeding programme efficiency (for example, with DNA marker assisted selection $[10])$, these programmes often remain reliant on iterations of backcrossing, gene pyramiding, and recurrent selection to yield desirable outputs [11]. Moreover, such an approach is liable to linkage drag, whereby untargeted introgression can cause deleterious effects [12].

However, the rise of genome editing (GE) technologies, particularly clustered regularly interspaced short palindromic repeat (CRISPR) based platforms is greatly increasing the speed and accuracy with which novel traits can be introduced into existing germplasm [11]. GE relies on programmable DNA nucleases, such as zinc-finger nucleases (ZFNs), transcriptional activator-like effector nucleases (TALENs) and CRISPR-crispr associated (CRISPR/Cas) complexes to introduce targeted double-strand breaks (DSBs) in DNA [13]. Both ZFNs and TALENS are protein guided tools, which makes them difficult to design, produce and validate, and has limited their widespread uptake in GE [14]. By contrast, CRISPR/Cas systems, being efficient, simple, and easily applied to a wide range of biological systems, have become widespread, and rapidly replaced ZFNs and TALENs in many laboratories [15].

The molecular mechanisms by which CRISPR/Cas GE functions have been extensively reviewed [16-21] and, therefore, will be described only briefly here. Naturally occurring CRISPRs (repetitive DNA sequences which are widespread in bacteria and archaea) contain many short sequences of viral origin, which are captured from invading pathogens and inserted into the genomic CRISPR regions [18]. Upon transcription, enzymatic processing yields short CRISPR-RNAs (crRNA), which associate with Cas endonuclease proteins to target complimentary invading DNA sequences of pathogens via hybridization. These DNA regions subsequently undergo double-strand cleavage. The CRISPR/Cas complex therefore acts as an RNA-guided adaptive immune system, able to provide resistance against viral pathogens upon secondary infection [20]. Notably (to avoid cleavage of the CRISPR genomic regions), the majority of CRISPR/Cas systems display a stringent requirement for a short, conserved sequence (known as the Protospacer Adjacent Motif -PAM) adjacent to the crRNA hybridization target for successful cleavage [22]. The most widely used of the CRISPR/Cas systems (type II) utilises a single Cas protein, Cas9, in conjunction with crRNA to query DNA targets for hybridization and subsequent cleavage (using either the HNH or RuvC nuclease domain in a strand-specific fashion) by the CRISPR/Cas9 complex [23]. This RNA-guided endonuclease system has been extensively repurposed for the goals of GE, and provision of Cas9 along with a single guide RNA (sgRNA) in cells is now a routine method for inducing DSBs in chosen genomic locations, including in bacteria, plants and human cells $[11,24-26]$. In plants, the introduction of a DSB triggers activation of the DNA repair machinery in targeted cells, which takes place via one of two mechanisms. The most common of these, operating throughout the duration of the cell cycle, is non-homologous end joining (NHEJ), resulting in indel mutations and loss of gene function. The second mechanism, homologous recombination (HR) is less common, but can be utilised with simultaneous provision of a homology-directed repair template for precise insertion or alteration of target loci $[27,28]$. Further to this, multiplexing of the CRISPR/Cas9 system has also been demonstrated in planta, whereby simultaneous provision of multiple sgRNAs guides Cas 9 proteins to cleave at multiple targeted genomic loci (for a comprehensive review see [29]). 
CRISPR/Cas GE can be used directly to introduce mutations into the germplasm of plant reproductive materials, producing novel traits independent of pre-existing genetic variation. Free from the linkage drag problem, these systems are revolutionising the improvement of both leafy and woody plants [11]. With the potential for multiplexed approaches [29] and transgene-free edited plants [30] CRISPR/Cas GE greatly accelerates the development of new crop cultivars. In this review, the theory and current methodologies for generating CRISPR-edited plants will be explored, and recurring challenges in this field addressed. The CRISPR-mediated improvement of leafy and woody plants will be focussed on from a disease-resistance perspective, with highlights from further areas of CRISPR application in abiotic stress tolerance also presented. The concluding section will cover future trends, including legislative regulation of CRISPR-edited crops and the emergence of Cpf1 and miRNA control as further tools for in planta GE.

\section{Theory of CRISPR-mediated GE in planta}

The initial step in a CRISPR/Cas GE pipeline is selection of a genomic target, and design of sgRNAs. To enable this, a well-annotated reference genome is desirable, as well as a software package for designing sgRNAs and minimising off-target effects. Native CRISPR/Cas9 in Streptococcus pyogenes uses a trans-acting crispr/crispr RNA (tracrRNA:crRNA duplex) to both promote maturation of the GE CRISPR/Cas complex, and guide the endonuclease Cas protein to its genomic target $[31,32]$. To permit facile $G E$, this native dual RNA system was engineered to form the sgRNA system now widely used with CRISPR/Cas GE, by creating a fusion tracrRNA:crRNA [32]. In order to target a genomic locus for editing, only the 20 nucleotides at the $5^{\prime}$ end of the sgRNA need be altered, such that the target site can be identified via complimentary base pairing $[17,32]$. For successful $G E$, the target site must be adjacent to a protospacer adjacent motif (PAM), a short sequence of DNA ( $5^{\prime}-\mathrm{NGG}-3^{\prime}$ for Cas9) specific to each type of Cas endonuclease [17]. In theory, therefore, sgRNA design requires knowledge of only a very short sequence of the target DNA, as well as the PAM specificity of the Cas endonuclease being used. However, without comprehensive knowledge of the whole genome sequence of the target organism, and potential redundancy in sgRNA target sites, off-target effects of CRISPR/Cas GE cannot be ruled out (see also 'recurring challenges for the application of CRISPR', below). Careful sgRNA design, accounting for potential off-target sites, is especially important, as mismatches between the sgRNA and the target site have been shown to be tolerated in CRISPR/Cas GE systems [17]. Multiple computational tools to aid sgRNA design now exist, including Desktop Genetics [33], sgRNA design rule based solutions [34], CRISPR focus [35], and some which have even begun to harness machine learning to improve performance, such as CRISTA [36], among others [36]. For a particularly comprehensive overview of computational tools see Demirci et al. (2017) [21]. sgRNA designs may then be synthesised and refined based on the user's requirements, cloning them under suitable promoters and transforming them into delivery vectors as required [30], or purifying them with a Cas endonuclease protein as ribonucleoproteins (RNPs), a technique which can subsequently allow DNA-free GE [38].

Delivery of the CRISPR/Cas apparatus required for GE can be achieved via one of several methods, depending on the desired outcome and developed methods for different plant species. The classical agrobacterium mediated plant transformation utilises a modified trans-kingdom DNA transfer chassis, avirulent Agrobacterium tumefaciens, to deliver the required genes for Cas protein and sgRNA expression into the host genome, from where they can be expressed [39]. As recovered transformants are hemizygous for the transgenes, selfing or backcrossing for $F 1$ segregation can result in transgene-free edited progeny $[11,30]$. Agroinfiltration can also be used to deliver CRISPR/Cas apparatus directly to leaves and uses injection or vacuum infiltration to introduce Agrobacterium cells directly into plant tissue. This method is not applicable to woody plants with open minor vein structures in their leaves [40].

Biolistic or 'gene-gun'- transformation can be used to deliver CRISPR/Cas encoding DNA or preassembled RNP complexes into plant cells, and is applicable to a wider range of plants than agrobacterium [41]. However, tissue regeneration and, if applicable, transgene performance, is influenced by biolistic particle size, acceleration, DNA amount and tissue type targeted, amongst other factors [42]. Precise tuning is required in each case of GE using biolistics to minimise damage to tissue and maximise the frequency of single copy transgene insertion events [43]. Protoplast transformation is a further method for introduction of CRISPR/Cas RNPs, and can be combined with biolistics or polyethylene glycol mediated transformation for GE $[44,45]$. Protoplasts are generated by enzymatic digestion of plant cell walls and can be used to regenerate whole plants. Whilst several plant species (mostly dicots) have proven amenable to protoplast isolation and regeneration [46], protocols for efficient protoplast usage in GE are still being developed for many crop plants, especially woody species $[30,44]$. 
Screening of plant cell cultures or regenerated transformants for verification of GE events can be carried out using multiple methods, including reporter gene activation [47], PCR screening [48], single strand conformation polymorphism analysis using PAGE [49], or sequencing of PCR amplicons [50] or whole genomes [47]. For a graphical workflow demonstrating this process, see figure 1.

CRISPR/Cas GE is not constrained to editing single targets, and multiplexing has become a common approach for editing multiple genetic loci simultaneously. Xie et al. (2015) made use of the host tRNA processing machinery in rice to express eight modified sgRNAs targeting four homologous genes from a single construct [51]. To the best of our knowledge, this remains the greatest number of target sites simultaneously edited in planta.

If CRISPR/Cas constructs are provided with an HDR template, homozygous pyramiding of chosen genes into the germplasm of elite cultivars can be achieved in one generation [52]. The HDR template copy number is often limiting in this process but, by using gemini viral based replicons for transient expression, HDR copy number has been boosted [53], and this approach has been successfully applied for HDR CRISPR/Cas GE in tomato, using agrobacterium-mediated transformation [54]. More recently, viral replicon based methods were combined with biolistic delivery for efficient transformation and HDR GE of wheat cells [55]. Systems have also been described whereby CRISPR/Cas constructs and repair templates are stably integrated into host genomes, with sgRNA hybridization targets added flanking the HDR templates. Therefore, on expression, the RNA guided endonuclease cuts its genomic target and releases the HDR template simultaneously, increasing the efficiency of site-specific repair template insertion [56]. 


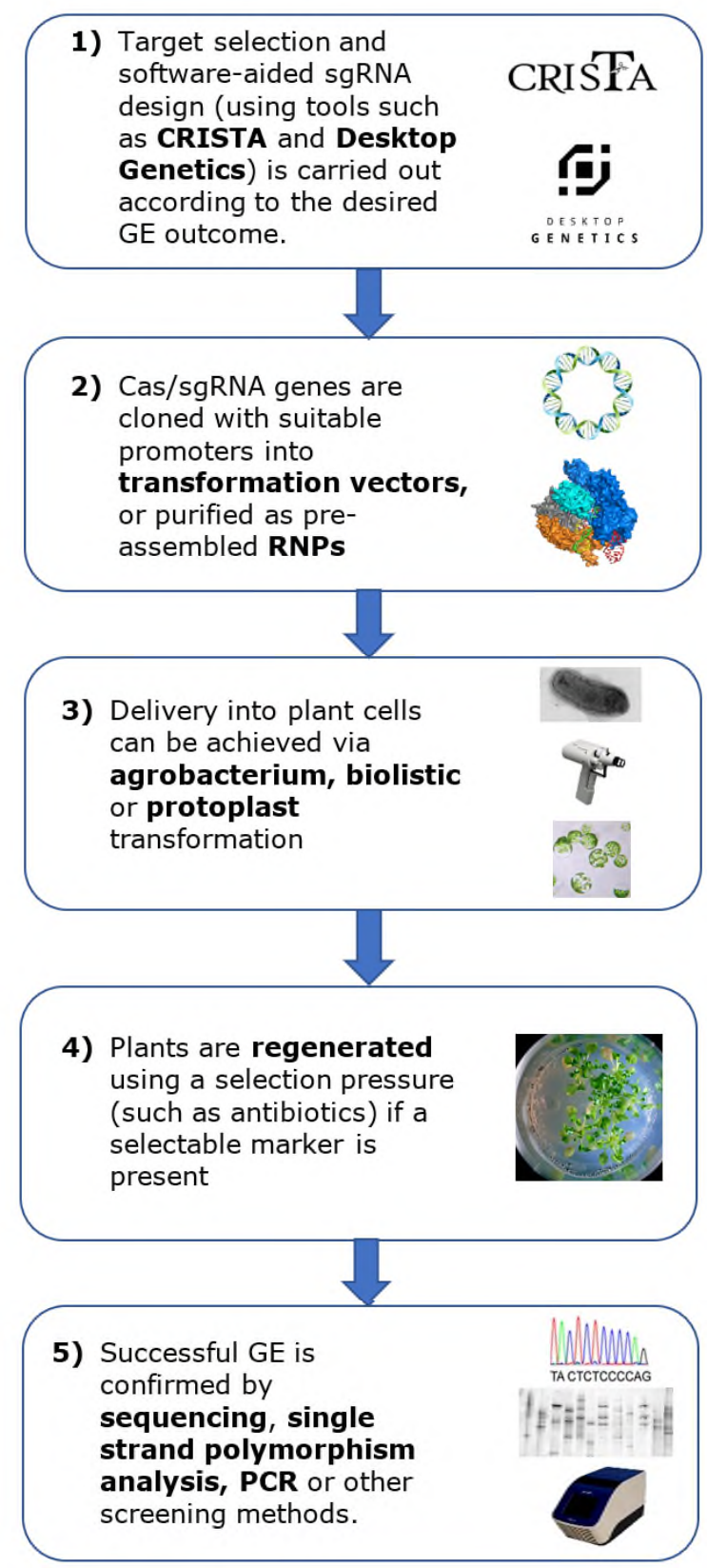

Figure 1. A workflow for in planta GE using CRISPR/Cas systems. The genome sequence of the target plant is used to identify GE target(s) from which computational tools can design sgRNAs to maximise efficiency and minimise offtarget effects (1). The sgRNA and Cas cassettes are cloned into expression vectors or expressed in vitro and isolated as RNPs (2). Transformation of plant cells using agrobacterium, protoplasts or biolistics delivers the CRISPR/Cas toolkit (3). Transformed cells can be regenerated into mature plants (4) and screened for successful GE events using a variety of methods (5). Note that step 5 may precede step 4 and, in some cases mature plants are not always regenerated. Text in bold corresponds to images illustrating each step, in corresponding order. 


\section{Recurring challenges for the application of CRISPR}

Common model organisms in plant research, including Arabidopsis, soybean and tomato, are selfing species possessing homozygous genomes [40]. However, in many flowering plants genome complexity is much greater due to larger size, heterozygosity, outcrossing and high frequency of SNPs [29]. Ingvarsson (2005) reported SNPs at one in every 60 base pairs for Populus tremula [57], and Külheim et al. (2009) reported a maximum frequency of one per 16 base pairs for eucalyptus [58]. Given these high rates of SNP occurrence, the need to account for polymorphisms in sgRNA design for GE is of great importance. However, many computational sgRNA design tools are limited in their application to outcrossing species, as the genome sequences used may lack biallelic or multiploid coverage [40]. Moreover, it has also been noted that in some cases (in poplar research, for example) the genotype subject to whole genome sequencing for use as a reference (Populus trichocarpa Nisqually-1) is different from the most commonly used genotype in genetic experiments, such as $P$. tremula $\mathrm{x}$ alba 717 [40]. To overcome these issues, Xue et al. (2015) developed a comprehensive variant-substituted custom genome for $P$. tremula $\mathrm{x}$ alba 717-1B4 using $\sim 20$-fold resequencing and public transcriptome data. This custom genome, sPta717, details $>10$ million SNPs and small indels in comparison to the $P$. trichocarpa version 3 reference genome, and is an example of a particularly useful tool for highly specific sgRNA design in poplar [59]. However, at the time of writing, such tools remain relatively rare for other outcrossing species. Of great concern in the use of CRISPR/Cas GE are off-target effects. Whilst it has been demonstrated that sgRNAs can tolerate multiple mismatches (such as those caused by SNPs), these can also lead to cleavage and mutagenesis of some non-target sequences [32,60-62]. However, several groups have demonstrated that the off-target problem can be overcome with careful sgRNA design. Zhou et al. (2015) analysed CRISPR/Cas off-target effects across duplicated genes in poplar. An sgRNA for the $4 C L 1$ gene was specifically designed to differentiate between $4 C L 1$ and the paralogous $4 C L 5$ gene. No off-target cleavage of the $4 C L 5$ gene was observed in the $4 C L 1$-edited transformants [40]. Using agrobacterium mediated transformation or agroinfiltration, the applicability of CRISPR/Cas GE has been demonstrated in multiple further woody plant species, in all cases successfully and with no off-target effects noted [3,63-66]. A summary of selected feasibility studies regarding CRISPR/Cas GE in woody plants can be seen in table 1, below.

As study by Jia et al. (2017) used CRISPR/Cas Ge to successfully disrupt CsLOB1 in citrus (see 'Applying CRISPR to the biotic stress problem: improving plants and trees', below). However the biological function of CSLOB1 in healthy plants throughout their lifecycle remains to be elucidated and, therefore, whilst no phenotypic changes were visible in the transformants (except citrus canker resistance), effects on later stage developmental processes (such as flowering) could not be discounted [67]. In plants such as citrus, where time to first flowering can be 2-3 years, there could be a developmental element in determining the overall success of a given GE approach, as well as genomic sequence and early phenotype elements. This problem is particularly pronounced in crops such as maize where, in 2016 , only $1 \%$ of genes had been functionally annotated with experimental data [68]. Functional genomics research in woody plants is made more challenging by long vegetative periods and low efficiencies in transformation $[3,69]$. Fortunately, genome sequences and annotation are likely to become more comprehensive with the increasing use of long-read and third-generation sequencing platforms [70]. However, the issue of functional experimental validation in plants with long juvenile phases is likely to remain for some time. Where off-target effects are of particular concern, a paired nickase strategy can be used. Here, the RuvC nuclease domain of Cas9 is inactivated via a D10A mutation. The remaining $\mathrm{HNH}$ domain introduces single strand breaks, repaired with high fidelity via base excision repair [71]. Therefore, to induce a mutagenic DSB, two nickases under strand specific sgRNAs must be delivered, targeting in close proximity [11]. This approach has been demonstrated in many plants, including Arabidopsis, rice and maize $[56,72-74]$.

A further challenge in CRISPR/Cas mediated plant GE is possible random integration of gene sequences and resulting GMO status of plants where genetic transformation is used to deliver the CRISPR/Cas components, such as with plasmid delivery via agrobacterium [45]. Whilst F0 plants generated in this way are GMOs, F1 segregation via selfing or back-crossing with the wild-type can remove these transgenes, leaving only the small indel in the target gene $[30,75]$. This is desirable, as such plants are less likely to be regulated as GMOs [76]. Where selfing is used, the F1 mutant retains the original traits of the starting cultivar, except for the editing event. However, where back-crossing is required (such as with fruit trees), the F1 offspring are likely to demonstrate large additional variability in comparison to the FO clone [30]. This problem, along with lengthy generation times for woody plants, represents a major challenge in improving woody plants. An alternative approach which circumvents these problems and is becoming more widely used is the delivery of CRISPR/Cas RNP complexes to protoplasts [45]. The RNPs induce GE events but are rapidly eliminated from the cell by endogenous protein degradation pathways, yielding a non-GMO 
edited protoplast [30]. Whilst this protoplast/RNP method presents clear advantages over those resulting in GMOs, regeneration of plants from protoplasts is still yet to be established in many woody plants $[46,77]$. 


\begin{tabular}{|c|c|c|c|c|c|}
\hline Plant Species & Target Gene & Methodology & Results/Traits & $\begin{array}{c}\text { Transgene } \\
\text { free? }\end{array}$ & Reference \\
\hline $\begin{array}{l}\text { Poplar (Populus } \\
\text { tomentosa) }\end{array}$ & PtoPDS & Agrobacterium mediated transformation & $\begin{array}{l}\text { Mutation efficiency of } 51.7 \% \text {, resulting in albino } \\
\text { phenotypes in regenerated plants and demonstrating } \\
\text { applicability of CRISPR/Cas GE in woody plants }\end{array}$ & No & [3] \\
\hline $\begin{array}{l}\text { Cotton } \\
\text { (Gossypium } \\
\text { hirsutum) - grows } \\
\text { as shrub or tree }\end{array}$ & $\begin{array}{l}\text { GhMYB25-like } A \\
\text { and GhMYB25-like } \\
D\end{array}$ & Agrobacterium mediated transformation & $\begin{array}{l}\text { High efficiency and specificity mutation of genes in } \\
\text { regenerated plants, demonstrating feasibility of } \\
\text { CRISPR/Cas GE in cotton }\end{array}$ & No & {$[63]$} \\
\hline $\begin{array}{l}\text { Poplar (Populus } \\
\text { tremula) }\end{array}$ & $4 C L 1$ & Agrobacterium mediated transformation & $\begin{array}{l}\text { Efficient mutation of } 4 C L 1 \text { in transformants, with no off- } \\
\text { target cleavage at the paralogous } 4 C L 5 \text { gene, providing } \\
\text { another proof of principle of CRISPR/Cas GE in woody } \\
\text { plants }\end{array}$ & No & {$[64]$} \\
\hline $\begin{array}{l}\text { Chardonnay grape } \\
\text { (Vitis vinefera) }\end{array}$ & $I d n D H$ & Agrobacterium mediated transformation & $\begin{array}{l}\text { A mutation efficiency of } 100 \% \text { in regenerated plants, } \\
\text { demonstrating the efficiency and specificity of CRISPR/Cas } \\
\text { GE in grape. }\end{array}$ & No & {$[65]$} \\
\hline $\begin{array}{l}\text { Sweet orange } \\
\text { (Citrus sinensis) }\end{array}$ & CSPDS & Leaf agroinfiltration & $\begin{array}{l}\text { CSPDS phytoene desaturase gene mutated in leaves at a } \\
\text { rate of } 3.2-3.9 \% \text { - proof of concept for agroinfiltration } \\
\text { CRISPR/Cas GE in orange }\end{array}$ & No & {$[66]$} \\
\hline
\end{tabular}

Table 1. Selected feasibility studies demonstrating CRISPR/Cas GE in woody plants 


\section{Applying CRISPR to the biotic stress problem: improving plants and trees}

CRISPR/Cas GE has been widely applied to different crop plants and some trees to enhance disease resistance, both by targeting endogenous genes and by transforming plants to express CRISPR/Cas constructs that directly target invading viruses (see tables 2 and 3 and also [21]). Different permutations of CRISPR/Cas GE have been used and, in some cases, transgene free, disease resistant cultivars have been isolated, with resistance targeting fungal, viral and bacterial pathogens (see tables 2 for leafy and 3 for woody plants).

Using the agrobacterium delivery method for transformation, Thomazella et al. (2016) introduced frameshift mutations in the targeted gene DMR6 in cotyledon segments of tomato. Regenerated plants had a broad disease resistance phenotype, including to the agriculturally relevant pathogens Pseudomonas syringae and Phytophthora capsici, although resistant plants remained transgenic, still carrying the agrobacterium-delivered vector DNA [78]. Wang et al. (2016) produced rice plants carrying indel mutations in the OsERF922 gene by using agrobacterium mediated transformation and, in contrast to Thomazella et al. (2016), were subsequently able to isolate transgene free plants via segregation in the T1 generation. These plants were resistant to rice blast disease, but were otherwise identical to non-edited plants [79]. Zhang et al. (2017) used biolistic plasmid delivery of CRISPR/Cas genes to transform wheat embryos, regenerating plants with resistance to powdery mildew, which were subject to segregation to remove transgenes [82]. Chandrasekaran et al. (2016) developed a virus resistant cucumber by using CRISPR/Cas9 to disrupt the recessive eIF4E gene, a eukaryotic translation initiation factor required to maintain the life cycle of many plant RNA viruses. Whilst successful eIF4E editing was observed, no off-target effects were seen in five putative off-target sites which were screened [80]. See table 2 for further examples of disease resistance generation via CRISPR/Cas GE in leafy plants.

As opposed to an agrobacterium or biolistic method, Malnoy et al. (2016) opted for a DNA-free engineering strategy in the woody crop plants apple and grape by delivering purified CRISPR/Cas RNPs to protoplasts. Successful mutagenesis was observed at all target sites across the apple and grape genomes, with no detectable off-target effects. Whilst the genes targeted (MLO-7 in grape and DIPM-1, 2, and 4 in apple) theoretically increase resistance to powdery mildew and fireblight respectively when mutated, plants were not regenerated from protoplasts in this study,

highlighting one of the challenges that often remains in woody plant transformation [45]. Jia et al. (2017) used CRISPR/Cas GE to disrupt CSLOB1 in citrus, a gene which can mediate susceptibility to citrus canker. Of seven potential off-target sites screened, no erroneous editing was observed in modified plants [67].

CRISPR/Cas GE has also seen application as a useful tool in poplar for reverse genetics. Wang et al. (2017) mutated the MYB115 gene in poplar, a transcriptional regulator of proanthocyanidin production, a collection of phenolic compounds important in plant defence. The MYB115 mutants showed increased susceptibility to the fungal pathogen Dothiorella gregaria, whereas poplar plants overexpressing this gene from a 35S promoter construct were resistant [88] (see also table 3). This study provides an elegant example of how CRISPR/Cas GE can be used to elucidate gene function and inform subsequent strategies to engineer plants for improvement.

These studies illustrate the diversity of CRISPR/Cas GE delivery methods and outcomes, and further recent studies demonstrating disease resistance generation via CRISPR/Cas GE in woody plants can be seen in table 3 . 


\begin{tabular}{|c|c|c|c|c|c|}
\hline $\begin{array}{l}\text { Tomato (Solanum } \\
\text { lycopersicum) }\end{array}$ & $D M R 6$ & $\begin{array}{l}\text { Agrobacterium mediated } \\
\text { transformation }\end{array}$ & $\begin{array}{l}\text { Broad disease, including to } P \text {. syringae, P.capsici and } \\
\text { Xanthomonas spp }\end{array}$ & No & [78] \\
\hline Rice (Oryza sativa) & OsERF922 & $\begin{array}{l}\text { Agrobacterium mediated } \\
\text { transformation }\end{array}$ & $\begin{array}{l}\text { Significantly enhanced resistance to rice blast, with no } \\
\text { change in agronomically relevant traits }\end{array}$ & Yes & {$[79]$} \\
\hline $\begin{array}{l}\text { Cucumber (Cucumis } \\
\text { sativus) }\end{array}$ & eIF4E & $\begin{array}{l}\text { Agrobacterium mediated } \\
\text { transformation }\end{array}$ & $\begin{array}{l}\text { Immunity to cucumber vein yellowing virus (Ipomovirus), } \\
\text { resistance to potyviruses zucchini yellow mosaic virus and } \\
\text { papaya ring spot mosaic virus-W }\end{array}$ & Yes & {$[80]$} \\
\hline $\begin{array}{l}\text { Tobacco (Nicotiana } \\
\text { benthamiana) }\end{array}$ & $\begin{array}{l}43 \text { loci in the beet } \\
\text { severe curly top virus } \\
\text { (BSCTV) genome }\end{array}$ & $\begin{array}{l}\text { Agrobacterium mediated } \\
\text { transformation - CRISPR/Cas and } \\
\text { viral targeted sgRNAs delivered }\end{array}$ & $\begin{array}{l}\text { No visible symptoms upon infection with BSCTV, virus } \\
\text { accumulation in leaves reduced } 90-97 \%\end{array}$ & No & {$[81]$} \\
\hline $\begin{array}{l}\text { Tobacco (Nicotiana } \\
\text { benthamiana) }\end{array}$ & $\begin{array}{l}6 \text { loci in the bean } \\
\text { yellow dwarf virus } \\
\text { (BeYDV) genome }\end{array}$ & $\begin{array}{l}\text { Agrobacterium mediated } \\
\text { transformation - CRISPR/Cas and } \\
\text { viral targeted sgRNAs delivered }\end{array}$ & $\begin{array}{l}\text { Reduced viral load and fewer symptoms on exposure to } \\
\text { BeYDV }\end{array}$ & No & {$[82]$} \\
\hline $\begin{array}{l}\text { Tobacco (Nicotiana } \\
\text { benthamiana) }\end{array}$ & $\begin{array}{l}3 \text { loci in the tomato } \\
\text { yellow leaf curl virus } \\
\text { (TYLCV) genome }\end{array}$ & $\begin{array}{l}\text { Agrobacterium mediated } \\
\text { transformation - CRISPR/Cas and } \\
\text { viral targeted sgRNAs delivered }\end{array}$ & $\begin{array}{l}\text { Reduced viral load and fewer symptoms on exposure to } \\
\text { TYLCV }\end{array}$ & No & [83] \\
\hline $\begin{array}{l}\text { Arabidopsis } \\
\text { (Arabidopsis thaliana) }\end{array}$ & eIF(iso) $4 E$ & $\begin{array}{l}\text { Agrobacterium mediated } \\
\text { transformation }\end{array}$ & $\begin{array}{l}\text { Complete resistance to turnip mosaic virus, with no effects } \\
\text { on plant vigour }\end{array}$ & Yes & {$[84]$} \\
\hline $\begin{array}{l}\text { Tomato (Solanum } \\
\text { lycopersicum) }\end{array}$ & SIMlo1 & $\begin{array}{l}\text { Agrobacterium mediated } \\
\text { transformation }\end{array}$ & $\begin{array}{l}\text { Complete resistance to powdery mildew, }<10 \text { months to } \\
\text { transgene free cultivar }\end{array}$ & Yes & {$[85]$} \\
\hline $\begin{array}{l}\text { Wheat (Triticum } \\
\text { aestivum L.) }\end{array}$ & $\begin{array}{l}3 \text { homoeologs of } \\
\text { EDR1 }\end{array}$ & Biolistic plasmid transformation & Resistance to powdery mildew & Yes & [86] \\
\hline
\end{tabular}

Table 2. Examples of CRISPR/Cas GE in leafy plants for disease resistance 


\section{Plant Species Target Gene}

Chardonnay grape

(Vitis vinefera)

and Golden

Delicious Apple

(Malus pumila)

Grapefruit (Citrus

paradisi

Macfadyen)

Wanjincheng

orange (Citrus

sinensis Osbeck)

Poplar (Populus

tomentosa)

$M L O-7$ in grape, DIPM-1, DIPM- 2 and DIPM-4 in

apple

CSLOB1

MYB115

\section{Methodology}

PEG-mediated RNP delivery to protoplasts

Efficient on-target editing of genes in protoplasts.

Theoretical powdery mildew (grape) and fireblight (apple) (protoplasts resistance, but plants not regenerated

Agrobacterium mediated transformation

Agrobacterium mediated transformation

Agrobacterium mediated transformation

Resistance to the citrus canker causative pathogen Xanthomonas citri subsp. citri

Increased susceptibility to Dothiorella gregaria fungus, only)

No

No

No

validating a MYB115 overexpression strategy to generate resistance

\section{Transgene Reference} free?

[45]

Table 3. Examples of CRISPR/Cas GE in woody plants for disease resistance 


\section{Further avenues for CRISPR: abiotic stress tolerance and plant production challenges}

In addition to generating disease resistance in crop plants, CRISPR/Cas GE has been widely applied to different challenges facing global plant production, including abiotic stresses such as drought, increasing yields and reducing time to harvest, among others [89-91].

For example, Shi et al. (2017) used CRISPR/Cas GE to tune expression of the ARGOS8 gene in maize, a negative regulator of ethylene responses. Using an HDR approach, they were able to replace the ARGOS8 promoter with a native maize GOS2 promoter, conferring moderate constitutive expression. Plants carrying the ARGOS8 variant showed increased yield in field trials under drought stress conditions, with no reduction in performance under non-stress conditions [89]. Osakabe et al. (2016) modified abiotic stress responses in Arabidopsis using a truncated gRNA-Cas9 system to generate site specific libraries of allelic variation for the OST2 gene, encoding a proton pump involved in stomatal closure. Plants carrying novel edited alleles exhibited enhanced stomatal closure upon heat stress [92], and this study highlights an important mechanism by which heat stress tolerant woody and leafy plants could be generated.

CRISPR/Cas GE is also being applied to target microRNAs (miRNAs, regulators of gene expression in plants) for the modulation of abiotic stress tolerance. Zhou et al. (2017) successfully targeted individual miRNA genes and whole miRNA gene families in rice at high efficiency in TO lines, demonstrating that OSMIR528 is an important positive regulator of the salt stress response [93]. Such knowledge forms an important basis for further investigations of the salt tolerance mechanisms in crop plants and could direct future engineering strategies for plant improvement. In pursuing yield improvements, $\mathrm{Li}$ et al (2016) targeted several yield related traits in rice in a single cultivar, using agrobacterium mediated transformation to deliver CRISPR/Cas components with sgRNA genes for multiple targets. By mutating Gn1a, DEP1 and GS3 in the Zhonghua II rice cultivar, regenerated plants showed an increase in grain size and number, as well as a more upright panicle architecture [90]. In a further yield-focussed study, Soyk et al. (2017) used CRSIPR/Cas GE to mutate SP5G in tomato, a gene with control over day-length sensitive flowering. Regenerated plants carrying SP5G mutations showed rapid flowering, a more compact growth phenotype, and shorter time to harvest [91]. Such work is of particular relevance to global food security, as compact growth habit crops could enable faster harvest and higher yields per unit area of agricultural land. Further CRISPR/Cas GE studies in tomato have also recently shown that it is possible to generate cultivars with increased shelf life [94] and, separately, parthenocarpic phenotype, able to confer greater stability in crop production under adverse conditions [95]. In addition to these studies, CRISPR/Cas GE has seen further application to challenges such as manipulating metabolic networks [96], enhancing fatty acid profiles of potential biofuel feedstocks [97] and even targeted alteration of flower colours [98].

\section{Conclusion and future perspectives}

With a rapidly increasing global population, changing climate and future agricultural uncertainties, new technologies to enhance food and resource security in plants are a global priority. CRISPR/Cas $\mathrm{GE}$, with its relative ease of use in relation to ZFNs and TALENS, variety of delivery methods, and capacity to generate null mutations in primary transformants, has seen rapid uptake in the field of woody and leafy plant improvement. Development of improved in silico tools for sgRNA design, vectors for CRISPR/Cas delivery and increasing volume of completed genome sequences enhances both the efficiency and accuracy of CRISPR/Cas GE systems.

These systems promise to rapidly enhance genetic improvement in woody plants, and the ability to multiplex editing, conduct homozygous pyramiding of desired genes into germplasm using HDR GE technology, and generate transgene free primary transformants will be of relevance to plants with lengthy juvenile phases.

Generation of transgene free edited plants is a particular advantage. The USDA's recent decisions not to regulate as GM a CRISPR edited mushroom and a 'waxy corn' cultivar [99] clearly indicates that CRISPR edited transgene free plants and trees will likely have cheaper, faster and broader routes to market than faced by 'traditional' GM crops.

Whilst a key challenge facing woody species in fast, uniform transgene free edited plant isolation remains the reliable regeneration of plants from edited protoplasts, the work of Malnoy et al. (2016) in grapevine and apple demonstrates important progress in illustrating the feasibility of this approach [45], and it seems likely that further development will be seen in the near future. As well as using CRISPR/Cas GE to target miRNA genes, Hirosawa et al. (2017) have demonstrated a miRNA responsive CRISPR/Cas GE system. By generating a synthetic, RNA based, miRNA controlled 'miR-Cas9' switch system, GE in human cell lines was modulated based on sensing of endogenous miRNA levels at the individual cell level [100]. This allows selective GE 
based on the individual transcriptional profiles of cells and could form a powerful tool if used to responsively control GE in planta in a cell type specific fashion.

Additionally, new developments in the use of CRISPR variants promise to deliver simpler and more predictable transgene free GE technologies in planta. Recently characterised CRISPR/Cpf1 (a type $\checkmark$ CRISPR effector) RNPs use shorter guide RNAs than Cas9 (facilitating simpler direct synthesis), induce larger target site deletions and show more favourable cleavage patterns for provided donor DNA insertion events [101]. Their use has been validated in lettuce protoplasts, and it is likely that Cpf1 will expand the toolbox of facile in planta GE technologies [101].

Given the rapid advances and widespread applications of CRISPR/Cas GE for plant and tree improvement, editing based breeding processes are likely to become more widespread for the rapid adaptation and improvement of cultivated species. By combining improving functional genomics knowledge, in silico tools and CRISPR/Cas GE systems with crop breeding schemes, the shift in breeding cycles will be toward one of targeted rounds of GE in high value germplasm for rapid adaptation and novel trait generation [11]. This pipeline looks set to replace some of the time-consuming crossing and untargeted mutagenesis cycles currently used in plant breeding. Increased use of CRISPR RNPs in protoplast engineering, permitting rapid generation of transgene free edited plants, seems particularly promising in the pursuit of improved leafy and woody plants with a clearer route from benchtop to field.

\section{Acknowledgements}

We thank CAB Reviews for the opportunity to write this paper. JP received financial support from the 1379 Society Old Member's Scholarship programme of New College, The University of Oxford, and the BBSRC. JM is support by Martin and Audrey Wood, and the Patsy Wood Trust.

\section{References}

1. Tilman D, Balzer C, Hill J, Befort BL. Global food demand and the sustainable intensification of agriculture. Proc Natl Acad Sci [Internet]. 2011;108(50):20260-4. Available from: http://www.pnas.org/cgi/doi/10.1073/pnas.1116437108

2. Osakabe Y, Sugano SS, Osakabe K. Genome engineering of woody plants: past, present and future. J Wood Sci. 2016;62(3):217-25.

3. Fan D, Liu T, Li C, Jiao B, Li S, Hou Y, et al. Efficient CRISPR/Cas9-mediated Targeted Mutagenesis in Populus in the First Generation. Sci Rep [Internet]. 2015;5(1):12217. Available from: http://www.nature.com/articles/srep12217

4. Ray DK, Mueller ND, West PC, Foley JA. Yield Trends Are Insufficient to Double Global Crop Production by 2050. PLoS One. 2013;8(6):1-8.

5. Bren d'Amour C, Reitsma F, Baiocchi G, Barthel S, Güneralp B, Erb K-H, et al. Future urban land expansion and implications for global croplands. Proc Natl Acad Sci [Internet]. 2016;114(34):201606036. Available from: http://www.pnas.org/lookup/doi/10.1073/pnas.1606036114

6. Piquerez SJM, Harvey SE, Beynon JL, Ntoukakis V. Improving crop disease resistance: lessons from research on Arabidopsis and tomato. Front Plant Sci [Internet].

2014;5(December):1-13. Available from: http://journal.frontiersin.org/article/10.3389/fpls.2014.00671/abstract

7. Bita CE, Gerats T. Plant tolerance to high temperature in a changing environment: scientific fundamentals and production of heat stress-tolerant crops. Front Plant Sci [Internet]. 2013;4(July):1-18. Available from: http://journal.frontiersin.org/article/10.3389/fpls.2013.00273/abstract

8. Zhu X-G, Long SP, Ort DR. Improving Photosynthetic Efficiency for Greater Yield. Annu Rev Plant Biol [Internet]. 2010;61(1):235-61. Available from:

http://www.ncbi.nlm.nih.gov/pubmed/20192734\%5Cnhttp://www.annualreviews.org/doi/1 0.1146/annurev-arplant-042809-112206

9. M. Perez-de-Castro A, Vilanova S, Canizares J, Pascual L, M. Blanca J, J. Diez M, et al. Application of Genomic Tools in Plant Breeding. Curr Genomics [Internet]. 2012;13(3):17995. Available from: http://www.eurekaselect.com/openurl/content.php?genre=article\&issn=13892029\&volume $=13 \&$ issue $=3 \&$ spage $=179$

10. Tester M, Langridge P. Breeding Technologies to Increase Crop Production in a Changing 
World. Science (80- ). 2010;327(5967):818-22.

11. Scheben A, Wolter F, Batley J, Puchta H, Edwards D. Towards CRISPR/Cas crops - bringing together genomics and genome editing. New Phytol. 2017;

12. Haggard JE, Johnson EB, St. Clair DA. Linkage Relationships Among Multiple QTL for Horticultural Traits and Late Blight ( P. infestans ) Resistance on Chromosome 5 Introgressed from Wild Tomato Solanum habrochaites. G3 Genes, Genomes, Genet [Internet]. 2013;3(12):2131-46. Available from: http://g3journal.org/lookup/doi/10.1534/g3.113.007195

13. Yin H, Kauffman KJ, Anderson DG. Delivery technologies for genome editing. Nat Rev Drug Discov [Internet]. 2017;16(6):387-99. Available from:

http://www.nature.com/doifinder/10.1038/nrd.2016.280

14. Ma D, Liu F. Genome Editing and Its Applications in Model Organisms. Genomics, Proteomics Bioinforma [Internet]. 2015;13(6):336-44. Available from:

http://dx.doi.org/10.1016/j.gpb.2015.12.001

15. Carroll D. A Perspective on the State of Genome Editing. Mol Ther [Internet]. $2016 ; 24(3): 412-3$. Available from:

http://www.ncbi.nlm.nih.gov/pubmed/26952917\%5Cnhttp://www.nature.com/mt/journal/v 24/n3/pdf/mt201628a.pdf

16. Ma Y, Zhang L, Huang X. Genome modification by CRISPR/Cas9. FEBS J. 2014;281(23):5186-93.

17. Doudna JA, Charpentier E. The new frontier of genome engineering with CRISPR-Cas9. Science (80- ) [Internet]. 2014;346(6213):1258096-1258096. Available from: http://www.sciencemag.org/content/346/6213/1258096.long

18. Sontheimer EJ, Barrangou R. The Bacterial Origins of the CRISPR Genome-Editing Revolution Downloaded. Hum Gene Ther. 2015;26(7):413-24.

19. Amitai G, Sorek R. CRISPR-Cas adaptation: insights into the mechanism of action. Nat Rev Microbiol [Internet]. 2016; advance on(2):67-76. Available from:

http://dx.doi.org/10.1038/nrmicro.2015.14

20. Jiang F, Doudna JA. CRISPR - Cas9 Structures and Mechanisms. AnnuRevBiophys [Internet]. 2017;46(March):505-29. Available from: www.annualreviews.org

21. Demirci Y, Zhang B, Unver T. CRISPR/Cas9: An RNA-guided highly precise synthetic tool for plant genome editing. J Cell Physiol. 2018;233(3):1844-59.

22. Karvelis T, Gasiunas G, Siksnys V. Harnessing the natural diversity and in vitro evolution of Cas9 to expand the genome editing toolbox. Curr Opin Microbiol [Internet]. 2017;37:8894. Available from: http://dx.doi.org/10.1016/j.mib.2017.05.009

23. Wang H, La Russa M, Qi LS. CRISPR/Cas9 in Genome Editing and Beyond. Annu Rev Biochem [Internet]. 2016;85(1):227-64. Available from: http://www.annualreviews.org/doi/10.1146/annurev-biochem-060815-014607

24. Doench JG, Hartenian E, Graham DB, Tothova Z, Hegde M, Smith I, et al. Rational design of highly active sgRNAs for CRISPR-Cas9-mediated gene inactivation. Nat Biotechnol

[Internet]. 2014;32(12):1262-7. Available from:

http://www.nature.com/doifinder/10.1038/nbt.3026

25. Barrangou R, Horvath P. A decade of discovery: CRISPR functions and applications. Nat Microbiol [Internet]. 2017;2(June):17092. Available from: http://www.nature.com/articles/nmicrobiol201792

26. Fogarty NME, McCarthy A, Snijders KE, Powell BE, Kubikova N, Blakeley P, et al. Genome editing reveals a role for OCT4 in human embryogenesis. Nature [Internet].

2017;550(7674):67-73. Available from:

http://www.nature.com/doifinder/10.1038/nature24033

27. Lin S, Staahl BT, Alla RK, Doudna JA. Enhanced homology-directed human genome engineering by controlled timing of CRISPR/Cas9 delivery. Elife. 2014;3:e04766.

28. Shen S, Loh TJ, Shen H, Zheng X, Shen H. CRISPR as a strong gene editing tool. BMB Rep [Internet]. 2016;50(September 2016):20-4. Available from:

http://www.ncbi.nlm.nih.gov/pubmed/27616359

29. Sattar MN, Iqbal Z, Tahir MN, Shahid MS, Khurshid M, Al-Khateeb AA, et al. CRISPR/Cas9: A Practical Approach in Date Palm Genome Editing. Front Plant Sci [Internet].

2017;8(August):1-16. Available from:

http://journal.frontiersin.org/article/10.3389/fpls.2017.01469/full 
30. Limera C, Sabbadini S, Sweet JB, Mezzetti B. New Biotechnological Tools for the Genetic Improvement of Major Woody Fruit Species. Front Plant Sci [Internet]. 2017;8(August):116. Available from: http://journal.frontiersin.org/article/10.3389/fpls.2017.01418/full

31. Deltcheva E, Chylinski K, Sharma CM, Gonzales K. CRISPR RNA maturation by trans encoded small RNA and host factor RNase III. Nature. 2011;471(7340):602-7.

32. Jinek M, Chylinski K, Fonfara I, Hauer M, Doudna JA, Charpentier E. A Programmable DualRNA - Guided DNA Endonuclease in Adaptive Bacterial Immunity. 2012;337(August):81622.

33. Hough SH, Ajetunmobi A, Brody L, Humphryes-Kirilov N, Perello E. Desktop Genetics. Per Med. 2016;13(6):517-21.

34. Doench JG, Fusi N, Sullender M, Hegde M, Vaimberg EW, Donovan KF, et al. Optimized sgRNA design to maximize activity and minimize off-target effects of CRISPR-Cas9. Nat Biotechnol [Internet]. 2016;34(2):184-91. Available from:

http://www.nature.com/doifinder/10.1038/nbt.3437

35. Cao Q, Ma J, Chen $\mathrm{CH}, \mathrm{Xu} \mathrm{H}$, Chen Z, Li W, et al. CRISPR-FOCUS: A web server for designing focused CRISPR screening experiments. PLoS One. 2017;12(9):1-8.

36. Abadi S, Yan WX, Amar D, Mayrose I. A machine learning approach for revealing patterns underlying CRISPR-Cas9 mechanism of action. PLoS Comput Biol. 2017;16(13):1-24.

37. Erard N, Knott SRV, Hannon GJ. A CRISPR Resource for Individual, Combinatorial, or Multiplexed Gene Knockout. Mol Cell [Internet]. 2017;67(2):348-354.e3. Available from: http://dx.doi.org/10.1016/j.molcel.2017.06.030

38. Woo JW, Kim J, Kwon S Il, Corvalán C, Cho SW, Kim H, et al. DNA-free genome editing in plants with preassembled CRISPR-Cas9 ribonucleoproteins. Nat Biotechnol [Internet]. 2015;33(11):1162-4. Available from: http://www.nature.com/doifinder/10.1038/nbt.3389

39. Char SN, Neelakandan AK, Nahampun H, Frame B, Main M, Spalding MH, et al. An Agrobacterium-delivered CRISPR/Cas9 system for high-frequency targeted mutagenesis in maize. Plant Biotechnol J. 2017;15(2):257-68.

40. Tsai C-J, Xue L-J. CRISPRing into the woods. GM Crops Food [Internet]. 2015;6(4):206-15. Available from: http://www.tandfonline.com/doi/full/10.1080/21645698.2015.1091553

41. Altpeter F, Baisakh N, Beachy R, Bock R, Capell T, Christou P, et al. Particle bombardment and the genetic enhancement of crops: Myths and realities. Mol Breed. 2005;15(3):305-27.

42. Liang Z, Chen K, Li T, Zhang Y, Wang Y, Zhao Q, et al. Efficient DNA-free genome editing of bread wheat using CRISPR/Cas9 ribonucleoprotein complexes. Nat Commun [Internet]. 2017;8(14261):1-5. Available from: http://www.nature.com/doifinder/10.1038/ncomms14261

43. Altpeter F, Springer NM, Bartley LE, Blechl A, Brutnell TP, Citovsky V, et al. Advancing Crop Transformation in the Era of Genome Editing. Plant Cell [Internet]. 2016;28(July):tpc.00196.2016. Available from: http://www.plantcell.org/lookup/doi/10.1105/tpc.16.00196

44. Svitashev S, Schwartz C, Lenderts B, Young JK, Mark Cigan A. Genome editing in maize directed by CRISPR-Cas9 ribonucleoprotein complexes. Nat Commun [Internet]. 2016;7:13274. Available from: http://www.nature.com/doifinder/10.1038/ncomms13274

45. Malnoy M, Viola R, Jung M-H, Koo O-J, Kim S, Kim J-S, et al. DNA-Free Genetically Edited Grapevine and Apple Protoplast Using CRISPR/Cas9 Ribonucleoproteins. Front Plant Sci [Internet]. 2016;7(December):1-9. Available from: http://journal.frontiersin.org/article/10.3389/fpls.2016.01904/full

46. Eeckhaut T, Lakshmanan PS, Deryckere D, Van Bockstaele E, Van Huylenbroeck J. Progress in plant protoplast research. Planta. 2013;238:991-1003.

47. Feng Z, Mao Y, Xu N, Zhang B, Wei P, Yang DL, et al. Multigeneration analysis reveals the inheritance, specificity, and patterns of CRISPR/Cas-induced gene modifications in Arabidopsis. Proc Natl Acad Sci U S A [Internet]. 2014;111(12):4632-7. Available from: http://www.ncbi.nlm.nih.gov/pubmed/24550464\%5Cnhttp://www.ncbi.nlm.nih.gov/pmc/ar ticles/PMC3970504/pdf/pnas.201400822.pdf

48. Xie K, Yang Y. RNA-Guided genome editing in plants using a CRISPR-Cas system. Mol Plant [Internet]. 2013;6(6):1975-83. Available from: http://dx.doi.org/10.1093/mp/sst119

49. Zhang $Y$, Liang Z, Zong $Y$, Wang $Y$, Liu J, Chen $K$, et al. Efficient and transgene-free genome editing in wheat through transient expression of CRISPR/Cas9 DNA or RNA. Nat Commun [Internet]. 2016;7(12617):1-8. Available from: 
http://www.nature.com/doifinder/10.1038/ncomms12617

50. Zhou H, Liu B, Weeks DP, Spalding MH, Yang B. Large chromosomal deletions and heritable small genetic changes induced by CRISPR/Cas9 in rice. Nucleic Acids Res.

2014;42(17):10903-14.

51. Xie K, Minkenberg B, Yang Y. Boosting CRISPR/Cas9 multiplex editing capability with the endogenous tRNA-processing system. Proc Natl Acad Sci [Internet]. 2015;112(11):3570-5. Available from: http://www.pnas.org/lookup/doi/10.1073/pnas.1420294112

52. Zhang H, Zhang J, Wei P, Zhang B, Gou F, Feng Z, et al. The CRISPR/Cas9 system produces specific and homozygous targeted gene editing in rice in one generation. Plant Biotechnol J. 2014;12(6):797-807.

53. Baltes NJ, Gil-Humanes J, Cermak T, Atkins PA, Voytas DF. DNA Replicons for Plant Genome Engineering. Plant Cell [Internet]. 2014;26(1):151-63. Available from: http://www.plantcell.org/cgi/doi/10.1105/tpc.113.119792

54. Čermák T, Baltes NJ, Čegan R, Zhang Y, Voytas DF. High-frequency, precise modification of the tomato genome. Genome Biol [Internet]. 2015;16(1):232. Available from: http://genomebiology.com/2015/16/1/232

55. Gil-Humanes J, Wang Y, Liang Z, Shan Q, Ozuna C V., Sánchez-León S, et al. Highefficiency gene targeting in hexaploid wheat using DNA replicons and CRISPR/Cas9. Plant J. 2017;89(6):1251-62.

56. Schiml S, Fauser F, Puchta H. The CRISPR/Cas system can be used as nuclease for in planta gene targeting and as paired nickases for directed mutagenesis in Arabidopsis resulting in heritable progeny. Plant J. 2014;80(6):1139-50.

57. Ingvarsson PK. Nucleotide polymorphism and linkage disequilibrium within and among natural populations of European aspen (Populus tremula L., salicaceae). Genetics. 2005;169(2):945-53.

58. Külheim C, Yeoh SH, Maintz J, Foley WJ, Moran GF. Comparative SNP diversity among four Eucalyptus species for genes from secondary metabolite biosynthetic pathways. BMC Genomics [Internet]. 2009;10(1):452. Available from: http://www.biomedcentral.com/1471-2164/10/452

59. Xue LJ, Alabady MS, Mohebbi M, Tsai CJ. Exploiting genome variation to improve nextgeneration sequencing data analysis and genome editing efficiency in Populus tremula $x$ alba 717-1B4. Tree Genet Genomes [Internet]. 2015;11(4). Available from: http://dx.doi.org/10.1007/s11295-015-0907-5

60. Ran FA, Hsu PD, Lin CY, Gootenberg JS, Konermann S, Trevino AE, et al. Double nicking by RNA-guided CRISPR cas9 for enhanced genome editing specificity. Cell [Internet]. 2013;154(6):1380-9. Available from: http://dx.doi.org/10.1016/j.cell.2013.08.021

61. Mali P, Aach J, Stranges PB, Esvelt KM, Moosburner M, Kosuri S, et al. CAS9 transcriptional activators for target specificity screening and paired nickases for cooperative genome engineering. Nat Biotechnol [Internet]. 2013;31(9):833-8. Available from: http://www.nature.com/doifinder/10.1038/nbt.2675

62. Pattanayak V, Lin S, Guilinger JP, Ma E, Doudna JA, Liu DR. High-throughput profiling of off-target DNA cleavage reveals RNA-programmed Cas9 nuclease specificity. Nat Biotechnol [Internet]. 2013;31(9):839-43. Available from: http://www.nature.com/doifinder/10.1038/nbt.2673

63. Li C, Unver T, Zhang B. A high-efficiency CRISPR/Cas9 system for targeted mutagenesis in Cotton (Gossypium hirsutum L.). Sci Rep [Internet]. 2017;7(43902):1-10. Available from: http://dx.doi.org/10.1038/srep43902

64. Zhou X, Jacobs TB, Xue LJ, Harding SA, Tsai CJ. Exploiting SNPs for biallelic CRISPR mutations in the outcrossing woody perennial Populus reveals 4-coumarate: CoA ligase specificity and redundancy. New Phytol. 2015;208(2):298-301.

65. Ren C, Liu X, Zhang Z, Wang Y, Duan W, Li S, et al. CRISPR/Cas9-mediated efficient targeted mutagenesis in Chardonnay (Vitis vinifera L.). Sci Rep [Internet]. 2016;6:1-9. Available from: http://dx.doi.org/10.1038/srep32289

66. Jia $\mathrm{H}$, Nian W. Targeted genome editing of sweet orange using Cas9/sgRNA. PLoS One. 2014;9(4):1-6.

67. Jia H, Zhang Y, Orbović V, Xu J, White FF, Jones JB, et al. Genome editing of the disease susceptibility gene CSLOB1 in citrus confers resistance to citrus canker. Plant Biotechnol J. 2017;15(7):817-23. 
68. Andorf CM, Cannon EK, Portwood JL, Gardiner JM, Harper LC, Schaeffer ML, et al. MaizeGDB update: New tools, data and interface for the maize model organism database. Nucleic Acids Res. 2016;44(D1):D1195-201.

69. Busov VB, Brunner AM, Meilan R, Filichkin S, Ganio L, Gandhi S, et al. Genetic transformation: A powerful tool for dissection of adaptive traits in trees. New Phytol. $2005 ; 167(1): 9-18$.

70. Yuan Y, Bayer PE, Batley J, Edwards D. Improvements in Genomic Technologies: Application to Crop Genomics. Trends Biotechnol [Internet]. 2017;35(6):547-58. Available from: http://dx.doi.org/10.1016/j.tibtech.2017.02.009

71. Fauser F, Schiml S, Puchta H. Both CRISPR/Cas-based nucleases and nickases can be used efficiently for genome engineering in Arabidopsis thaliana. Plant J. 2014;79(2):348-59.

72. Schiml S, Fauser F, Puchta H. Repair of adjacent single-strand breaks is often accompanied by the formation of tandem sequence duplications in plant genomes. Proc Natl Acad Sci [Internet]. 2016;113(26):7266-71. Available from: http://www.pnas.org/lookup/doi/10.1073/pnas.1603823113

73. Mikami M, Toki S, Endo M. Precision Targeted Mutagenesis via Cas9 Paired Nickases in Rice. Plant Cell Physiol. 2016;57(5):1058-68.

74. Wolter F, Edelmann S, Kadri A, Scholten S. Characterization of paired Cas9 nickases induced mutations in maize mesophyll protoplasts. Maydica. 2017;62:1-11.

75. Jones HD. Regulatory uncertainty over genome editing. Nat Plants [Internet]. 2015;1(1):14011. Available from: http://www.nature.com/articles/nplants201411

76. Waltz E. CRISPR-edited crops free to enter market, skip regulation. Nat Biotechnol [Internet]. 2016;34(6):582-582. Available from:

http://www.nature.com/doifinder/10.1038/nbt0616-582

77. Mezzetti B, Landi L, Phan BH, Taruschio L, Lim KY. Peg-mediated fusion of rubus idaeus (Raspberry) and R. fruticosus (blackberry) protoplasts, selection and characterisation of callus lines. Plant Biosyst. 2001;135(1):63-9.

78. de Toledo Thomazella DP, Brail Q, Dahlbeck D, Staskawicz BJ. CRISPR-Cas9 mediated mutagenesis of a DMR6 ortholog in tomato confers broad-spectrum disease resistance. bioRxiv [Internet]. 2016;64824. Available from:

http://biorxiv.org/lookup/doi/10.1101/064824

79. Wang F, Wang C, Liu P, Lei C, Hao W, Gao Y, et al. Enhanced rice blast resistance by CRISPR/ Cas9-Targeted mutagenesis of the ERF transcription factor gene OsERF922. PLoS One. 2016;11(4):1-18.

80. Chandrasekaran J, Brumin M, Wolf D, Leibman D, Klap C, Pearlsman M, et al. Development of broad virus resistance in non-transgenic cucumber using CRISPR/Cas9 technology. Mol Plant Pathol. 2016;17(7):1140-53.

81. Ji X, Zhang H, Zhang Y, Wang Y, Gao C. Establishing a CRISPR-Cas-like immune system conferring DNA virus resistance in plants. Nat Plants [Internet]. 2015;1(10):15144.

Available from: http://www.nature.com/articles/nplants2015144

82. Baltes NJ, Hummel AW, Konecna E, Cegan R, Bruns AN, Bisaro DM, et al. Conferring resistance to geminiviruses with the CRISPR-Cas prokaryotic immune system. Nat Plants [Internet]. 2015;1(10):15145. Available from: http://www.nature.com/articles/nplants2015145

83. Ali Z, Abulfaraj A, Idris A, Ali S, Tashkandi M, Mahfouz MM. CRISPR/Cas9-mediated viral interference in plants. Genome Biol [Internet]. 2015;16(1):238. Available from: http://genomebiology.com/2015/16/1/238

84. Pyott DE, Sheehan E, Molnar A. Engineering of CRISPR/Cas9-mediated potyvirus resistance in transgene-free Arabidopsis plants. Mol Plant Pathol. 2016;17(8):1276-88.

85. Nekrasov V, Wang C, Win J, Lanz C, Weigel D, Kamoun S. Rapid generation of a transgenefree powdery mildew resistant tomato by genome deletion. Sci Rep [Internet]. 2017;7(1):482. Available from: http://www.nature.com/articles/s41598-017-00578-x

86. Zhang Y, Bai Y, Wu G, Zou S, Chen Y, Gao C, et al. Simultaneous modification of three homoeologs of TaEDR1 by genome editing enhances powdery mildew resistance in wheat. Plant J. 2017;91(4):714-24.

87. Peng A, Chen S, Lei T, Xu L, He Y, Wu L, et al. Engineering canker-resistant plants through CRISPR/Cas9-targeted editing of the susceptibility gene CsLOB1 promoter in citrus. Plant Biotechnol J. 2017;1-11. 
88. Wang L, Ran L, Hou Y, Tian Q, Li C, Liu R, et al. The transcription factor MYB115 contributes to the regulation of proanthocyanidin biosynthesis and enhances fungal resistance in poplar. New Phytol. 2017;215(1):351-67.

89. Shi J, Gao H, Wang H, Lafitte HR, Archibald RL, Yang M, et al. ARGOS8 variants generated by CRISPR-Cas9 improve maize grain yield under field drought stress conditions. Plant Biotechnol J. 2017;15(2):207-16.

90. Li M, Li X, Zhou Z, Wu P, Fang M, Pan X, et al. Reassessment of the Four Yield-related Genes Gn1a, DEP1, GS3, and IPA1 in Rice Using a CRISPR/Cas9 System. Front Plant Sci [Internet]. 2016;7(March):1-13. Available from: http://journal.frontiersin.org/Article/10.3389/fpls.2016.00377/abstract

91. Soyk S, Müller NA, Park SJ, Schmalenbach I, Jiang K, Hayama R, et al. Variation in the flowering gene SELF PRUNING 5G promotes day-neutrality and early yield in tomato. Nat Genet [Internet]. 2017;49(1):162-8. Available from:

http://www.nature.com/doifinder/10.1038/ng.3733

92. Osakabe Y, Watanabe T, Sugano SS, Ueta R, Ishihara R, Shinozaki K, et al. Optimization of CRISPR/Cas9 genome editing to modify abiotic stress responses in plants. Sci Rep [Internet]. 2016;6(1):26685. Available from: http://www.nature.com/articles/srep26685

93. Zhou J, Deng K, Cheng Y, Zhong Z, Tian L, Tang X, et al. CRISPR-Cas9 Based Genome Editing Reveals New Insights into MicroRNA Function and Regulation in Rice. Front Plant Sci [Internet]. 2017;8(1598):1-12. Available from: http://journal.frontiersin.org/article/10.3389/fpls.2017.01598/full

94. Yu Q, Wang B, Li N, Tang Y, Yang S, Yang T, et al. CRISPR / Cas9-induced Targeted Mutagenesis and Gene Replacement to Generate Long-shelf Life Tomato Lines. Sci Rep [Internet]. 2017;(2014721023):1-18. Available from: http://dx.doi.org/10.1038/s41598017-12262-1

95. Ueta R, Abe C, Watanabe T, Sugano SS, Ishihara R, Ezura H, et al. Rapid breeding of parthenocarpic tomato plants using CRISPR/Cas9. Sci Rep [Internet]. 2017;7(1):507. Available from: http://www.nature.com/articles/s41598-017-00501-4

96. Li R, Li R, Li X, Fu D, Zhu B, Tian H, et al. Multiplexed CRISPR/Cas9-mediated metabolic engineering of $\mathrm{Y}$-aminobutyric acid levels in Solanum lycopersicum. Plant Biotechnol J. 2017;1-13.

97. Jiang WZ, Henry IM, Lynagh PG, Comai L, Cahoon EB, Weeks DP. Significant enhancement of fatty acid composition in seeds of the allohexaploid, Camelina sativa, using CRISPR/Cas9 gene editing. Plant Biotechnol J. 2017;15(5):648-57.

98. Watanabe K, Kobayashi A, Endo M, Sage-Ono K, Toki S, Ono M. CRISPR/Cas9-mediated mutagenesis of the dihydroflavonol-4-reductase-B (DFR-B) locus in the Japanese morning glory Ipomoea (Pharbitis) nil. Sci Rep [Internet]. 2017;7(1):10028. Available from: http://www.nature.com/articles/s41598-017-10715-1

99. Waltz E. Gene-edited CRISPR mushroom escapes US regulation. Nature. 2016;532(September):293.

100. Hirosawa M, Fujita Y, Parr CJC, Hayashi K, Kashida S, Hotta A, et al. Cell-type-specific genome editing with a microRNA-responsive CRISPR-Cas9 switch. Nucleic Acids Res. 2017;45(13).

101. Kim H, Kim S-T, Ryu J, Kang B-C, Kim J-S, Kim S-G. CRISPR/Cpf1-mediated DNA-free plant genome editing. Nat Commun [Internet]. 2017;8:14406. Available from: http://www.nature.com/doifinder/10.1038/ncomms14406 